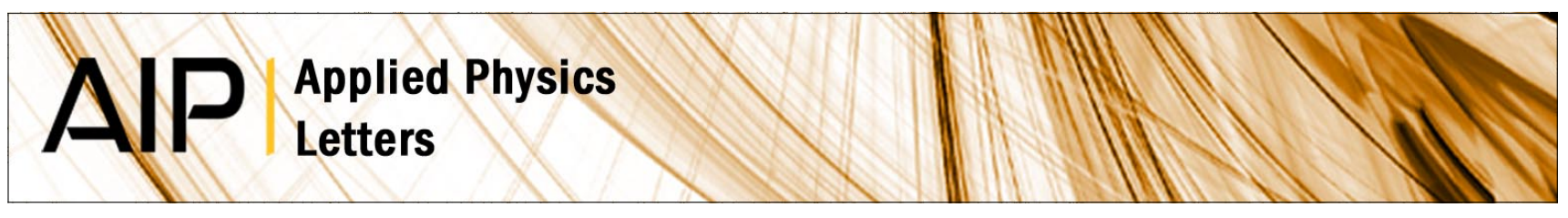

\title{
Unipolar behavior of asymmetrically doped strained Si0.5Ge0.5 tunneling field-effect transistors
}

M. Schmidt, R. A. Minamisawa, S. Richter, A. Schäfer, D. Buca et al.

Citation: Appl. Phys. Lett. 101, 123501 (2012); doi: 10.1063/1.4751356

View online: http://dx.doi.org/10.1063/1.4751356

View Table of Contents: http://apl.aip.org/resource/1/APPLAB/v101/i12

Published by the American Institute of Physics.

Additional information on Appl. Phys. Lett.

Journal Homepage: http://apl.aip.org/

Journal Information: http://apl.aip.org/about/about_the_journal

Top downloads: http://apl.aip.org/features/most_downloaded

Information for Authors: http://apl.aip.org/authors

\section{ADVERTISEMENT}

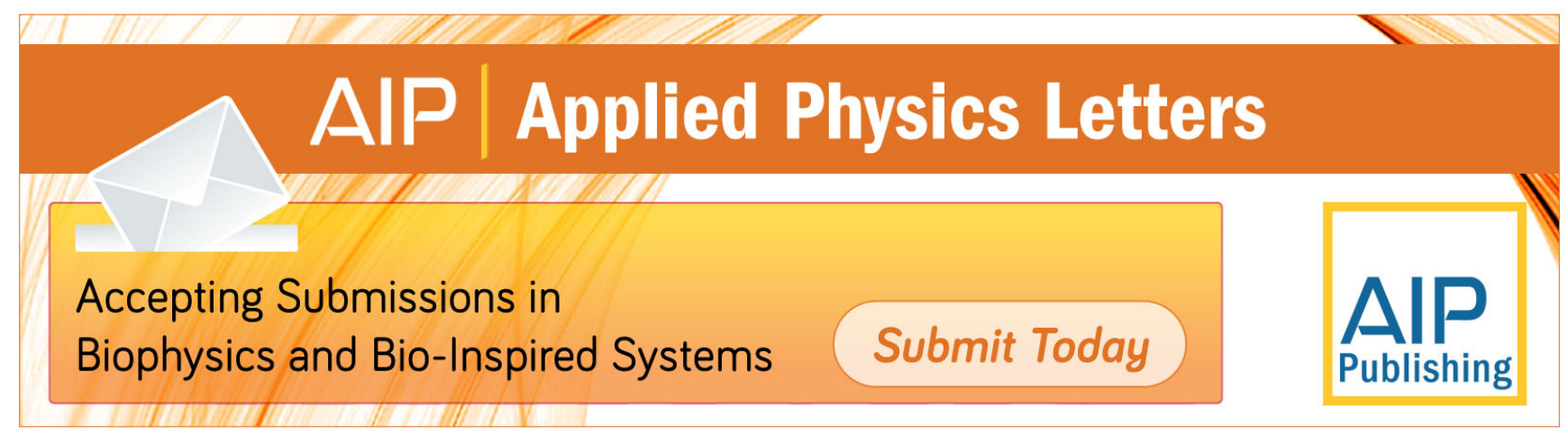




\title{
Unipolar behavior of asymmetrically doped strained $\mathrm{Si}_{0.5} \mathrm{Ge}_{0.5}$ tunneling field-effect transistors
}

\author{
M. Schmidt, ${ }^{1}$ R. A. Minamisawa, ${ }^{1}$ S. Richter, ${ }^{1}$ A. Schäfer, ${ }^{1}$ D. Buca,,${ }^{1}$ J. M. Hartmann, ${ }^{2}$ \\ Q.-T. Zhao, ${ }^{1, a)}$ and S. Mant1 ${ }^{1}$ \\ ${ }^{1}$ Peter Grünberg Institute 9 (PGI9-IT) and JARA-Fundamentals of Future Information Technologies, \\ Forschungszentrum Jülich, 52425 Jülich, Germany \\ ${ }^{2}$ CEA-LETI, MINATEC Campus, 17 rue des Martyrs, 38054 Grenoble, France
}

(Received 11 July 2012; accepted 24 August 2012; published online 17 September 2012)

\begin{abstract}
We investigate here the impact of the dopant concentration in the source and drain regions on the ambipolar behavior of band-to-band tunneling field-effect transistors with compressively strained $\mathrm{Si}_{0.5} \mathrm{Ge}_{0.5}$ channels grown on $\mathrm{Si}$ on insulator. Source and drain areas were formed by $\mathrm{BF}_{2}{ }^{+}$and $\mathrm{As}^{+}$ion implantation to doses of $1 \times 10^{13}, 1 \times 10^{14}$, and $1 \times 10^{15} \mathrm{~cm}^{-2}$. We show that the dopant concentration impacts the energy band alignment of source/drain and the channel region, and thus influences the tunneling current. The ambipolar device behavior is strongly reduced toward unipolar for source-to-drain implantation dose ratio of 100, but at the expense of the on-current, as compared to symmetric implanted devices. Moreover, our results indicate that for SiGe devices, the change of the B doping concentration has a greater impact on the tunneling currents than the variation of the As concentration. (C) 2012 American Institute of Physics. [http://dx.doi.org/10.1063/1.4751356]
\end{abstract}

During the past decades, there has been a growing interest in developing device concepts which comply with the requirements of low power consumption. The concept of the band-to-band tunneling field-effect transistor (TFET) with its potential for sub- $60 \mathrm{mV} /$ decade subthreshold swing, ${ }^{1}$ a prerequisite for scaling the supply voltage well below $1 \mathrm{~V}$, are of great interest for low power applications. ${ }^{2,3}$ Furthermore, the TFETs present reduced short-channel effects compared to metal-oxide-semiconductor field-effect transistors (MOSFETs). Several materials and device architectures such as carbon nanotubes (CNT), III-V semiconductors, or Ge and Si nanowires were proved to improve TFET device performance. $^{4-6}$ Inter-band tunneling probability depends largely on the semiconductor band-gap, $E_{\mathrm{g}}$, and the maximum electric field at the junction. Silicon-germanium $\left(\mathrm{Si}_{1-x} \mathrm{Ge}_{x}\right)$ is a very promising candidate, allowing band-gap adjustment via the Ge content, $x$, and the elastic strain, $\varepsilon$, and presents lower effective masses and higher mobilities of holes than $\mathrm{Si}^{7}$ Recently, planar strained SiGe tunneling FETs with superior drive currents compared to $\mathrm{Si}$ were reported. ${ }^{8}$ A disadvantage of conventional TFETs is, however, the ambipolar transport behavior. In such devices, the minority carrier tunneling occurs at both the $p^{+}-i$ ( $n$-TFET) or the $n^{+}-i$ ( $p$-TFET) junction, depending on the channel energy band edges relative to the source or drain energy band positions.

The most known method for suppressing ambipolar behavior is employing two semiconducting materials with different band-gap for source/gate and drain, where the band offset disables the tunneling effect in the drain. ${ }^{9}$ However, the fabrication of such hetero-structure devices is very challenging and involves critical processing steps control such as perfect growth compatibility of the semiconductor layers and highly selective etching. ${ }^{10}$

\footnotetext{
${ }^{\text {a) }}$ Author to whom correspondence should be addressed. Electronic mail: q.zhao@fz-juelich.de.
}

In this letter, we study the influence of the B and As dopant concentration on the tunneling efficiency at the $p^{+}-i$ and $n^{+}-i$ junctions of TFETs. The aim is to determine the optimum combination of asymmetrical $p$ - and $n$-type doping in order to reduce the TFETs ambipolar characteristics, towards a unipolar behavior. The devices are fabricated on compressively strained $\mathrm{Si}_{0.5} \mathrm{Ge}_{0.5}$ and feature a $\mathrm{HfO}_{2} / \mathrm{TiN}$ gate stack. We interpret our results in terms of dopant dynamic, in order to elucidate the role of diffusion and activation mechanisms on the band structure of the tunneling strained SiGe device. ${ }^{11}$ Dopant diffusion in such structures is particularly complex since it is dependent on both strain and Ge content. ${ }^{12}$

TFETs were fabricated on $12 \mathrm{~nm}$ thin $\mathrm{Si}_{0.5} \mathrm{Ge}_{0.5}$ layers pseudomorphically grown on $10 \mathrm{~nm}$ silicon-on-insulator (SOI) substrates by reduced pressure chemical vapor deposition (RP-CVD). ${ }^{13}$ The SiGe layers were capped with $3 \mathrm{~nm} \mathrm{Si}$ in order to maintain the high quality of the Si/high- $k$ interface. The pseudomorphic SiGe layers are biaxially compressively strained to $\varepsilon=-2.1 \%$ as confirmed by Raman and $\mathrm{x}$-ray diffraction (XRD) analyses. The fabricated devices are isolated to each other by mesa etching. The gate stack with a length of $5 \mu \mathrm{m}$ is formed with $5 \mathrm{~nm} \mathrm{HfO} 2$ dielectric film and $15 \mathrm{~nm}$ TiN metal gate.

Symmetrical doped devices which operate as both, $n$ and $p$-TFETs, were fabricated using $\mathrm{As}^{+}$and $\mathrm{BF}_{2}^{+}$ion implantation at source/drain (S/D) with the same dose of $10^{15} \mathrm{~cm}^{-2}$ and used as comparison reference. In this study "symmetrical doping" refers to equal implantation dose, not to equal activation of dopant concentration. To study the influence of the doping asymmetry, $n / p$-TFET devices were processed keeping the $\mathrm{BF}_{2}^{+} / \mathrm{As}^{+}$dose at source to $10^{15} \mathrm{~cm}^{-2}$, whereas the $\mathrm{As}^{+} / \mathrm{BF}_{2}{ }^{+}$drain implantation doses were decreased to $10^{14}$ and $10^{13} \mathrm{~cm}^{-2}$, respectively. The use of $\mathrm{BF}_{2}^{+}$ion implantation ions conserves the elastic strain within the pseudomorphic SiGe layer, assures reasonable dopant activation while the fluorine atoms decrease the $\mathrm{B}$ diffusivity. ${ }^{14}$ For all devices, the $\mathrm{As}^{+}$and 
$\mathrm{BF}_{2}{ }^{+}$implantation energies were $4.2 \mathrm{keV}$ and $2.7 \mathrm{keV}$, respectively. A critical parameter for TFETs is the steepness of the tunneling junctions in the SiGe. In this respect, the activation was performed at a moderate temperature of $650^{\circ} \mathrm{C}$ for $1 \mathrm{~min}$.

The TFET with symmetrical S/D implantation exhibits typical ambipolar behavior (Fig. 1(a), black curve). $n$-TFETs built on $\mathrm{SiGe}$ show a better performance compared to $p$-TFETs, which has been previously reported in several works. ${ }^{6,15}$ Hence, we will mainly focus on the $n$-TFET for the discussion of the experimental results, although the $p$-TFET behaves analogously (Fig. 2). A positive applied gate voltage $V_{\mathrm{gg}}$ leads to an energy alignment of the occupied states in the heavily $p$-doped source with the empty energy levels in the intrinsic channel (bands overlapping) (see Fig. 1(c)). The tunneling current is then given by

$$
I_{D}=\frac{q}{h} \int_{E_{C}^{C h}}^{E_{V}^{S}} T(E)\left(\mu_{S}-\mu_{D}\right) d E,
$$

where $T(E)$ is the tunneling probability, $\mu_{\mathrm{S}}$ and $\mu_{\mathrm{D}}$ are the Fermi levels at source and drain, and $E_{\mathrm{V}} \mathrm{S}$ and $E_{\mathrm{C}}{ }^{\mathrm{Ch}}$ are the

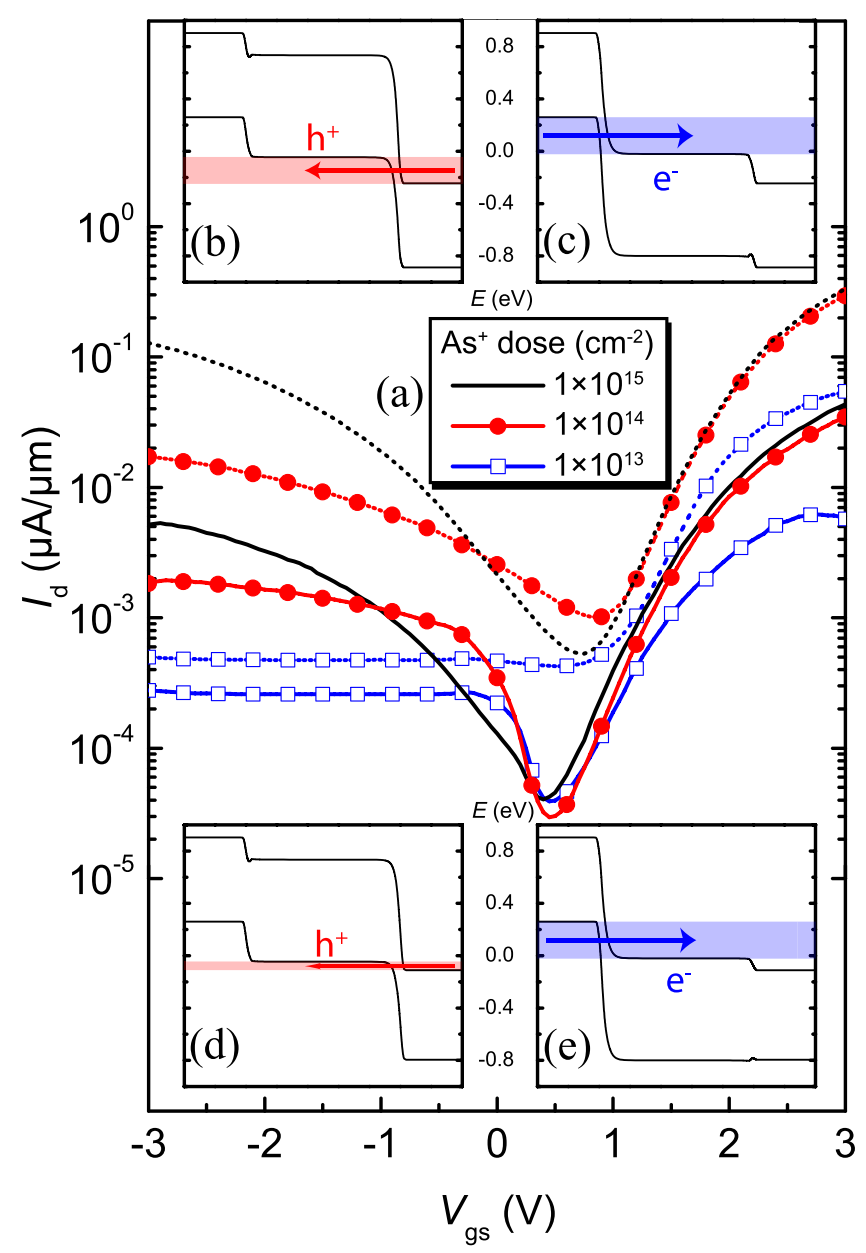

FIG. 1. (a) Transfer characteristics of $\mathrm{Si}_{0.5} \mathrm{Ge}_{0.5} n$-TFETs with S/D implantations of $1 \times 10^{15} \mathrm{BF}_{2}^{+} / \mathrm{cm}^{2}\left[1 \times 10^{15}\right.$ (black), $1 \times 10^{14}$ (red full circles), and $1 \times 10^{13} \mathrm{As}^{+} / \mathrm{cm}^{2}$ (blue empty squares), respectively]. Solid curves are for $V_{\mathrm{ds}}=0.5 \mathrm{~V}$ and dotted for $V_{\mathrm{ds}}=1.7 \mathrm{~V}$. (b)-(e) The simulated band structure displayed in the insets indicates the influence for the drain dopant concentration on the tunneling current: (b) and (c) $N_{\mathrm{A}}=N_{\mathrm{D}}=2 \times 10^{20} \mathrm{~cm}^{-3}$; (d) and (e) $N_{\mathrm{A}}=2 \times 10^{20} \mathrm{~cm}^{-3}, N_{\mathrm{D}}=1 \times 10^{19} \mathrm{~cm}^{-3}$. The bias conditions are (b) and (d) $V_{\mathrm{ds}}=0.1 V_{\mathrm{gs}}=-0.5$ and (c) and (e) $V_{\mathrm{ds}}=0.1, V_{\mathrm{gs}}=-0.5$.

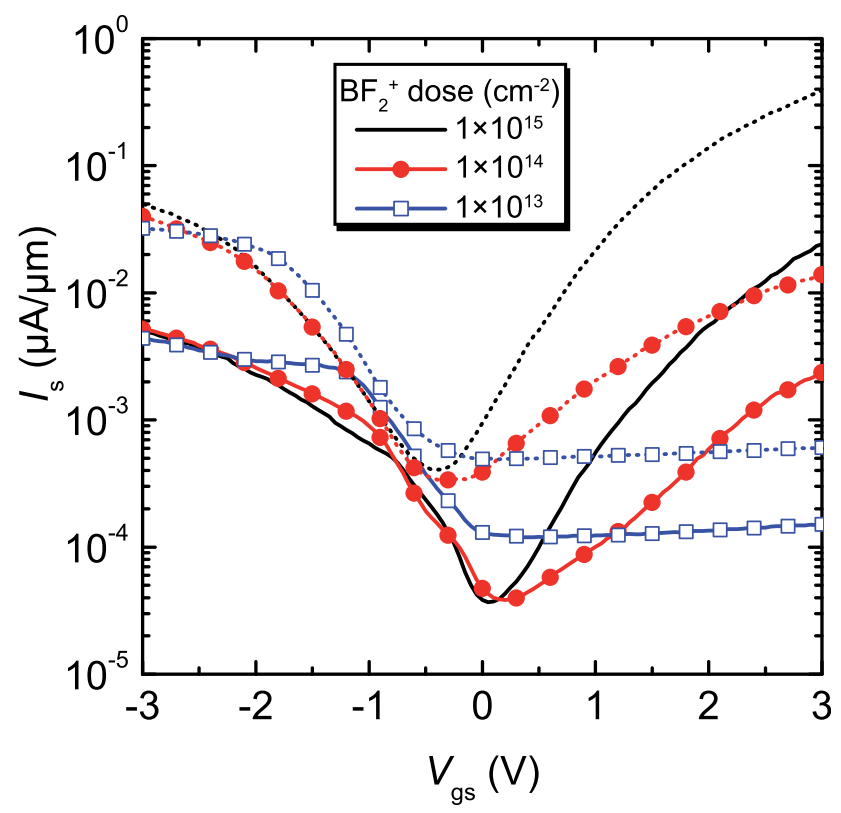

FIG. 2. Transfer characteristics of $\mathrm{Si}_{0.5} \mathrm{Ge}_{0.5} p$-TFETs with S/D implantations of $1 \times 10^{15} \mathrm{As}^{+} / \mathrm{cm}^{2}\left[1 \times 10^{15}\right.$ (solid and dotted black lines), $1 \times 10^{14}$ (red full circles), and $1 \times 10^{13} \mathrm{BF}_{2}^{+} / \mathrm{cm}^{2}$ (blue empty circles), respectively]. Solid curves are for $V_{\mathrm{ds}}=0.5 \mathrm{~V}$ and dotted for $V_{\mathrm{ds}}=1.7 \mathrm{~V}$.

valence and the conduction band edges at the tunneling source and the channel, respectively. For negative gate voltages (reverse branch), $V_{\mathrm{gs}}<0$, where tunneling occurs at the drain/channel junction (Fig. 1(c)), the current decreases with the $\mathrm{As}^{+}$implantation dose. For a B ${ }^{+} / \mathrm{As}^{+}$dose ratio of 10:1 (Fig. 1(a), red full circles), the reverse branch is significantly lowered, and for a ratio of 100:1 (Fig. 1(a), blue empty squares). The reverse current is suppressed to the off-state value. A negative $V_{\mathrm{gs}}$ potential leads to hole tunneling from the $n$-doped drain into the channel (Fig. 1(b)), and the resulting tunneling current can be estimated analogously to Eq. (1). The energy of the Fermi levels in source, $\mu_{\mathrm{S}}$, and drain, $\mu_{\mathrm{D}}$, are given by the concentrations of acceptors $N_{\mathrm{A}}$ and donors $N_{\mathrm{D}}$, respectively. Since the Fermi levels-e.g., $\mu_{\mathrm{D}}$ - are pinned by the (inner) potential, a decrease of $N_{\mathrm{D}}$ in the drain up-shifts the energy bands leading to a smaller overlap and a decreased tunnel current (Fig. 1(d)). On the source side, where the $N_{\mathrm{A}}$ is kept constant, the tunneling probability at the source side is unaffected (see Fig. 1(e)). The tunnel-barrier width decrease with the gate voltage directly results in an increase of the on-current as long as the dominant resistance is contributed by the tunnel barrier, with the channel resistance being only a fraction of the tunnelbarrier resistance. The degradation of the on-currents observed for higher $V_{\mathrm{gs}}$ is ascribed to an increased drain resistance due to decreased dopant concentration, which starts dominating the source and channel resistance.

From the discussion above, we conclude that the TFETs performance is strongly related with the S/D junctions' properties: steep junctions allow a better electrostatic control, while high doping level defines the S/D resistances. Ultrashallow junction formation in $\mathrm{Si}$ was intensively studied for both $p$ - and $n$-type, but only recently studies on $p$-type junction formation on SiGe were also reported. ${ }^{16}$ However, there is still a lack on $n$-type doping studies of SiGe materials. 


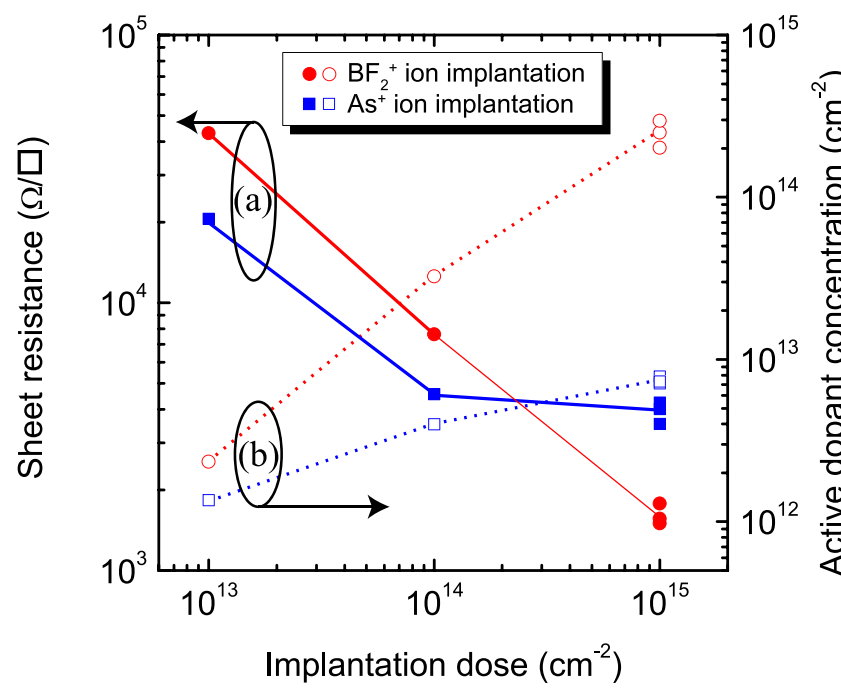

FIG. 3. (a) Active dopant dose and (b) sheet resistance vs. ion implantation dose.

Sheet resistance and active dopant concentration acquired by Hall measurements for $\mathrm{BF}_{2}{ }^{+}$and $\mathrm{As}^{+}$doped $\mathrm{Si}_{0.5} \mathrm{Ge}_{0.5}$ test samples are shown in Figs. 3(a) and 3(b), respectively. The implantation and annealing parameters are identical with the one used for TFET fabrication. The sheet resistance of the doped layers can be described as a power law $\rho \sim N^{\alpha}$, where $N$ is the sheet dopant concentration, with $\alpha=-1.09$ for $\mathrm{B}$ and $\alpha=-0.76$ for As. The exponent for $\mathrm{B}$ is in good agreement with the theoretical value $\alpha_{\text {theor }}=-1$ (Ref. 17) but the exponent for As deviates greatly from $\alpha_{\text {theor }}$, due to low activation of As atoms. Another important issue is the exponential increase of the Schottky resistance, which will become significantly high for lower values of $N$. The total resistance for a TFET is given by

$$
R_{\mathrm{tot}}=R_{\mathrm{tunnel}}+R_{\text {channel }}+R_{\mathrm{S} / \mathrm{D}}
$$

For high dopant concentrations on the drain side $N>10^{19} \mathrm{~cm}^{-3}$, the Schottky contact resistance is negligible, $R_{\text {tunnel }} \gg R_{\mathrm{S} / \mathrm{D}}+R_{\text {channel }}$. For lower dopant concentrations $\left(N<10^{18} \mathrm{~cm}^{-3}\right)$, the Schottky contact resistance of the metalsemiconductor contact becomes comparable to the tunneling resistance. ${ }^{18}$ Hence, the increase of $R_{\text {tot }}$ leads to smaller oncurrents, even if the tunnel junction on the source side is unaffected.

In order to trade-off between the desired suppression of tunneling from drain to channel, and the undesired reduction of $I_{\mathrm{on}}$ due to S/D resistance, we define $I_{\mathrm{n}}=I\left(V_{\mathrm{gs}, \min }+\Delta V\right)$ as a measurement for $I_{\mathrm{on}}$, and $I_{\mathrm{p}}=I\left(V_{\mathrm{gs}, \min }-\Delta V\right)$ as a measurement for $I_{\text {off. }} \Delta V$ is a parameter chosen in a way that it can be compared to $V_{\mathrm{t}}$ of a MOSFET. In contrast to the latter one, the TFET does not feature a comparable threshold and thus lacks a $V_{\mathrm{t}}$, though attempts to define such parameter have been discussed. ${ }^{19}$ In this work, we chose $\Delta V$ to be $2 \mathrm{~V}$ and $I\left(V_{\mathrm{gs}, \min }\right)$ is the minimum current. In Table $\mathrm{I}, I_{\mathrm{n}}$ and $I_{\mathrm{p}}$ are presented for all three $n$-TFETs and for low $\left(V_{\mathrm{ds}}=0.5 \mathrm{~V}\right)$ and high $\left(V_{\mathrm{ds}}=1.7 \mathrm{~V}\right)$ drain voltages. For $V_{\mathrm{ds}}=0.5 \mathrm{~V}$, and an As dose of $10^{14} \mathrm{~cm}^{-2}, I_{\mathrm{n}}$ is $76 \%$ from the value of the symmetrically implanted device, while $I_{\mathrm{p}}$ reduces to $31 \%$. The As dose of $10^{13} \mathrm{~cm}^{-2}$ leads to a further $I_{\mathrm{n}}$ decrease
TABLE I. $I_{\mathrm{n}}$ and $I_{\mathrm{p}}$ values of $\mathrm{Si}_{0.5} \mathrm{Ge}_{0.5}$ TFETs at drain voltages of $V_{\mathrm{ds}}=0.5 \mathrm{~V}$ and $1.7 \mathrm{~V}$ and for different source to drain ion implantations ratio. The current values were averaged over 180 devices.

\begin{tabular}{llll}
\hline \hline$V_{\mathrm{ds}}(\mathrm{V})$ & $\mathrm{BF}_{2}{ }^{+} / \mathrm{As}^{+}$ & $I_{\mathrm{n}}(\mu \mathrm{A} / \mu \mathrm{m})$ & $I_{\mathrm{p}}(\mu \mathrm{A} / \mu \mathrm{m})$ \\
\hline & $100: 100$ & $2.23 \times 10^{-2}$ & $2.21 \times 10^{-3}$ \\
& $100: 10$ & $1.61 \times 10^{-2}$ & $6.77 \times 10^{-4}$ \\
0.5 & $100: 1$ & $5.24 \times 10^{-3}$ & $2.21 \times 10^{-4}$ \\
& $100: 100$ & $2.04 \times 10^{-1}$ & $3.66 \times 10^{-2}$ \\
& $100: 10$ & $2.12 \times 10^{-1}$ & $4.77 \times 10^{-3}$ \\
1.7 & $100: 1$ & $3.27 \times 10^{-2}$ & $4.27 \times 10^{-4}$ \\
\hline \hline
\end{tabular}

down to $30 \%$ of the symmetrically device, while $I_{\mathrm{p}}$ to $10 \%$. At higher drain voltages, e.g., $V_{\mathrm{ds}}=1.7 \mathrm{~V}$, this picture is even clearer. $I_{\mathrm{n}}$ is almost the same for the As dose of $10^{14} \mathrm{~cm}^{-2}$ as it is for the dose of $10^{15} \mathrm{~cm}^{-2}$ (also see in Fig. 1(a)), but decreased to $16 \%$ for $10^{13} \mathrm{~cm}^{-2}$. The $I_{\mathrm{p}}$ drive current is reduced to $13 \%$ and $1 \%$, respectively. For device performance, an As dose of $10^{14} \mathrm{~cm}^{-2}$ would be favorable, because it leads to a clearly suppressed $I_{\mathrm{p}}$ branch, while the decrease of $I_{\mathrm{n}}$ is arguably small.

In conclusion, $n$ - and $p$-TFET devices with symmetrically doped source and drain show a distinct ambipolar behavior. We demonstrate that employing asymmetrically doped source and drain regions is a simple and efficient modality of reducing this ambipolarity. $n$-TFETs with decreased As dose exhibit significantly lower reverse currents due to reduced band overlap at the $n^{+}-i$ junction, while $p$-TFETs with decreased $\mathrm{B}$ dose behave analogously. The on-currents of the devices are decreased, caused by an increase of the Schottky contact resistance at the drain side. Hall measurements showed that the variation of the B implantation dose over three decades largely impacts the acceptor concentration $N_{\mathrm{A}}$, whereas the variation of the As implantation dose has a minor influence on $N_{\mathrm{D}}$, and consequently on the contact resistance. We show that there is an optimum drain-tosource doping ration which led to a highly decreased reverse tunneling current, while the forward tunneling current is insignificantly reduced.

This work was supported by the European project STEEPER.

${ }^{1}$ J. Appenzeller, Y.-M. Lin, J. Knoch, and P. Avouris, Phys. Rev. Lett. 93, $1-4$ (2004).

${ }^{2}$ Y. Khatami and K. Banerjee, IEEE Trans. Electron Devices 56, 27522761 (2009)

${ }^{3}$ A. M. Ionescu and H. Riel, Nature 479, 329-337 (2011).

${ }^{4}$ W. Y. Choi, B. Park, J. D. Lee, and T. K. Liu, IEEE Electron Device Lett. 28, 743-745 (2007).

${ }^{5}$ Q. Zhang, S. Sutar, T. H. Kosel, and A. C. Seabaugh, Solid-State Electron. 53, 30-35 (2009).

${ }^{6}$ J. T. Smith, C. Sandow, S. Das, R. A. Minamisawa, S. Mantl, and J. Appenzeller, IEEE Trans. Electron Devices 58, 1822-1829 (2011).

${ }^{7}$ R. A. Minamisawa, M. Schmidt, L. Knoll, D. Buca, Q. T. Zhao, J. M. Hartmann, K. K. Bourdelle, and S. Mantl, IEEE Electron Device Lett. 33, 1105-1107 (2012).

${ }^{8}$ M. Schmidt, R. A. Minamisawa, S. Richter, R. Luptak, J. M. Hartmann, D. Buca, Q. T. Zhao, and S. Mantl, Solid-State Electron. 71, 42-47 (2012).

${ }^{9}$ D. Kazazis, P. Jannaty, A. Zaslavsky, C. Le Royer, C. Tabone, L. Clavelier, and S. Cristoloveanu, Appl. Phys. Lett. 94, 263508 (2009).

${ }^{10}$ G. K. Chang, T. K. Carns, S. S. Rhee, and K. L. Wang, J. Electrochem. Soc. 138, 202 (1991). 
${ }^{11}$ K. Rajendran and W. Schoenmaker, J. Appl. Phys. 89, 980 (2001).

${ }^{12}$ P. Delugas and V. Fiorentini, Phys. Rev. B 69, 1-5 (2004).

${ }^{13}$ J. M. Hartmann, A. Abbadie, and S. Favier, J. Appl. Phys. 110, 083529 (2011).

${ }^{14}$ R. A. Minamisawa, D. Buca, B. Holländer, J. M. Hartmann, K. K. Bourdelle, and S. Mantl, J. Electrochem. Soc. 159, H44 (2012).

${ }^{15}$ O. M. Nayfeh, J. L. Hoyt, and D. A. Antoniadis, IEEE Trans. Electron Devices 56, 2264-2269 (2009).
${ }^{16}$ R. A. Minamisawa, M. Schmidt, E. Durgun Özben, J. M. J. Lopes, J. M. Hartmann, K. K. Bourdelle, J. Schubert, Q. T. Zhao, D. Buca, and S. Mantl, Microelectron. Eng. 88, 2955-2958 (2011).

${ }^{17}$ S. M. Sze and K. K. Ng, Physics of Semiconductor Devices, 3rd ed. (John Wiley \& Sons, Inc., New York, 2007).

${ }^{18}$ A. Y. C. Yu, Solid-State Electron. 13, 239-247 (1970).

${ }^{19}$ K. Boucart and A. M. Ionescu, Solid-State Electron. 52, 1318-1323 (2008). 